\title{
Vortices and Quasiparticles near the Superconductor-Insulator Transition in Thin Films
}

\author{
Victor M. Galitski, ${ }^{1}$ G. Refael, ${ }^{1}$ Matthew P. A. Fisher, ${ }^{1}$ and T. Senthil ${ }^{2,3}$ \\ ${ }^{1}$ Kavli Institute for Theoretical Physics, University of California, Santa Barbara, California 93106-4030, USA \\ ${ }^{2}$ Center for Condensed Matter Theory, Indian Institute of Science, Bangalore-560012, India \\ ${ }^{3}$ Department of Physics, Massachusetts Institute of Technology, Cambridge, Massachusetts 02139, USA
}

(Received 9 May 2005; published 9 August 2005)

\begin{abstract}
We study the low temperature behavior of an amorphous superconducting film driven normal by a perpendicular magnetic-field $(B)$. For this purpose we introduce a new two-fluid formulation consisting of fermionized field-induced vortices and electrically neutralized Bogoliubov quasiparticles (spinons) interacting via a long-ranged statistical interaction. This approach allows us to access a novel nonFermi-liquid phase, which naturally interpolates between the low $B$ superconductor and the high $B$ normal metal. We discuss the properties of the resulting "vortex metal" phase.
\end{abstract}

DOI: 10.1103/PhysRevLett.95.077002

Superconductivity in two dimensions (2D) provides a unique area to explore a fascinating variety of quantum phenomena. Of particular interest are strongly disordered superconducting films, tuned by a perpendicular magnetic field into the normal state. Early experiments [1-3] focused on a magnetic-field tuned "superconductorinsulator" transition at a critical field $B_{\mathrm{c}}$. Based on a phenomenological "dirty-boson" model [4], universal scaling behavior in the temperature and field dependence of the resistance was predicted near the quantum phase transition $\left(B \approx B_{\mathrm{c}}\right)$ separating the superconductor from the Bose insulator. Although some experimental support was found for scaling of $R(T, B)$, the resistance at the separatrix, $R\left(T=0, B_{\mathrm{c}}\right)$ was often found to deviate significantly from the expected universal value (near $R_{Q}=h / 4 e^{2}$ ).

More recent experiments on such amorphous films at lower temperatures revealed a rather rich and surprising behavior in $R(T \rightarrow 0, B)$. Specifically, for temperatures well below the mean-field transition where Cooper pairs are well formed, the resistance saturates at a value which can be many orders of magnitude smaller than the normal state resistance $[5,6]$. This mysterious strange metal phase occurs over a reasonably large range of intermediate fields, $B \leq B_{\mathrm{c}}$. Moreover, in highly disordered films with $B_{\mathrm{c}} \ll$ $H_{\mathrm{c} 2}$, the resistance at higher fields was found to exhibit a dramatic nonmonotonic dependence, rising in some cases up to values of order $10^{6} R_{Q}$, before plummeting towards the normal state resistance at $B \approx H_{\mathrm{c} 2}$ [7-9]. A bosononly theory that might exhibit a metallic phase was proposed by Dalidovic and Phillips [10]. Kapitulnik et al. [11],
PACS numbers: 74.78.-w, 73.43.Nq, 74.20.De

however, argued for the importance of fermionic quasiparticles ignored in the dirty-boson approach.

In this Letter we develop a two-fluid formulation, which incorporates fermionic quasiparticles into the dirty-boson model. The two fluids consist of field-induced vortices and electrically neutralized quasiparticles ("spinons") interacting via a long-ranged statistical interaction. In the low temperature limit the vortices must be treated as quantum particles and their statistics will be important. Previous work has implicitly assumed that the vortices are bosonic. However in the presence of unpaired electronic excitations the issue of vortex statistics can be subtle. Here, we explore the consequences of treating the vortices as fermions (see also [12]). Technically this is achieved via a Chern-Simons flux attachment to the bosonic vortices. With fermionic vortices and fermionic electronlike quasiparticles, our twofluid model can be studied within an RPA approximation. The fermionized vortices can quantum diffuse, leading to a description of a novel conducting non-Fermi-liquid phase which interpolates between the low field superconductor and the high field normal state. We suggest that this vortex metal phase might account for the strange metallic behavior observed in InO and MoGe films. Moreover, our twofluid approach gives a natural explanation for the nonmonotonic dependence of the low temperature magnetoresistance. Below we discuss transport, thermodynamic, and tunneling properties of the vortex metal.

Consider a 2D disordered superconductor in a perpendicular magnetic field below the mean-field BCS upper critical field $H_{\mathrm{c} 2}$. For concreteness we consider a lattice tight-binding Hamiltonian of an $s$-wave superconductor,

$$
\mathcal{H}=-t \sum_{\left\langle\mathbf{r} \mathbf{r}^{\prime}\right\rangle}\left[c_{\mathbf{r} \alpha}^{\dagger} c_{\mathbf{r}^{\prime} \alpha} e^{i \mathbf{A}_{\mathbf{r r}^{\prime}}}+\text { H.c. }\right]+\frac{1}{2 C} \sum_{\mathbf{r}}\left(2 n_{\mathbf{r}}+c_{\mathbf{r} \alpha}^{\dagger} c_{\mathbf{r} \alpha}-n_{0}\right)^{2}+\Delta \sum_{\mathbf{r}}\left[e^{i \phi_{\mathbf{r}}} c_{\mathbf{r} \uparrow} c_{\mathbf{r} \downarrow}+\text { H.c. }\right]+\mathcal{H}_{\text {disorder }},
$$

where $\Delta$ is the magnitude of the superconducting order parameter, $C^{-1}$ is an on-site charging energy, and $n_{0}$ is the electrical charge density. At temperatures well below the mean-field transition, it is necessary to include quantum fluctuations of the Cooper pairs, which were incorporated phenomenologically via the phase of the superconducting order parameter, $\phi_{\mathbf{r}}$, which is conjugate to the Cooper pair number operator, $n_{\mathbf{r}}$. Because of the external magnetic field and strong disorder, one expects a substantial number of low-energy electrons will also be present, certainly in the vortex cores and perhaps elsewhere. Moreover, since the strange metal is resistive, the vortices are mobile, and a correct description will 
likely require incorporating the quantum dynamics of both vortices and fermionic quasiparticles.

Following Refs. [13,14] it is convenient to make a change of variables: $f_{\mathbf{r} \alpha}^{\dagger}=b_{\mathbf{r}} c_{\mathbf{r} \alpha}^{\dagger}$, where $\left(b_{\mathbf{r}}^{\dagger}\right)^{2}=e^{i \phi_{\mathbf{r}}}$ creates a Cooper pair at site $\mathbf{r}$. This transformation removes the electric charge from the quasiparticles, leaving charge zero and spin one-half fermionic spinons, $f_{\mathbf{r}}$. The use of spinon variables is merely a technical convenience and does not necessarily imply that they are the good excitations in the system. Indeed in the vortex metal phase the spin-carrying excitations will ultimately be electrons (not spinons). Next, a duality transformation can be implemented which exchanges the operators $b_{\mathbf{r}}$ for $h c / 2 e$ vortices, leading to a theory of bosonic vortices in a fluctuating gauge field which mediates the intervortex long-range interactions. Moreover, the vortices and spinons have a statistical interaction between them; i.e., a vortex "sees" a spinon as a source of a $\pi$ flux and vice versa.

We describe the vortex-spinon mixture with a so-called $U(1)$ formulation [14,15]. In the long-wavelength lowenergy limit the corresponding Euclidean action is $S=$ $\int d \mathbf{x} d \tau\left[\mathcal{L}_{\mathrm{v}}+\mathcal{L}_{\mathrm{s}}+\mathcal{L}_{\text {int }}\right]$, where $\mathcal{L}_{\mathrm{v}}$ is the vortex Lagrangian in terms of the vortex bosonic fields $\Psi$ and the gauge field $a_{\mu}=\left(\mathbf{a}, a_{0}\right)=\left(a_{x}, a_{y}, a_{0}\right)$,

$$
\mathcal{L}_{\mathrm{v}}=\Psi^{\dagger}\left[-\frac{(-i \mathbf{a}+i \alpha)^{2}}{2 m_{\mathrm{v}}}+\left(\partial_{\tau}-i a_{0}+i \alpha_{0}\right)\right] \Psi+\mathcal{L}_{a},
$$

with $\mathcal{L}_{a}=\frac{1}{2 C}\left(\epsilon_{\mu \nu \lambda} \partial_{\nu} a_{\lambda}-\delta_{\mu \tau} \pi n_{0}\right)^{2}$. The spinon's action is

$$
\mathcal{L}_{\mathrm{s}}=f_{\alpha}^{\dagger}\left[-\frac{1}{2 m_{\mathrm{s}}}(\nabla-i \beta)^{2}+\left(\partial_{\tau}-i \beta_{0}\right)\right] f_{\alpha},
$$

and the vortex-spinon statistical interaction is mediated by two auxiliary $U(1)$ fields $\alpha_{\mu}$ and $\beta_{\mu}$,

$$
\mathcal{L}_{\text {int }}=-(i / \pi) \epsilon_{\mu \nu \lambda} \alpha_{\mu} \partial_{\nu} \beta_{\lambda} .
$$

The equations of motion $\delta \mathcal{L} / \delta \alpha_{0}=0$ and $\delta \mathcal{L} / \delta \beta_{0}=0$ attach $\pi$ flux to the vortices and spinons, $\epsilon_{i j} \partial_{i} \beta_{j}=\pi \hat{N}_{\mathrm{v}}=$ $\pi \Psi^{\dagger} \Psi$, and $\epsilon_{i j} \partial_{i} \alpha_{j}=\pi \hat{n}_{\mathrm{f}}=\pi f_{\alpha}^{\dagger} f_{\alpha}$, with $\hat{N}_{\mathrm{v}}$ and $\hat{n}_{\mathrm{f}}$ the vortex and spinon densities. The total electrical charge density is given by $\hat{n}_{0}=\left(\epsilon_{i j} \partial_{i} a_{j}\right) / \pi$, and for small capacitance will be set by the $c$ number $n_{0}$, that is $\left\langle\hat{n}_{0}\right\rangle=n_{0}$. The average number of vortices is set by the external magnetic field $B$ through $\pi\left\langle N_{\mathrm{v}}\right\rangle=B(\hbar=c=e=1)$.

In the strange metal, where the film resistance $R(T)$ saturates at low temperatures, it appears that the vortices are diffusing. To access this within the vortex-spinon theory, it will prove extremely convenient to statistically transmute the vortices, converting them into fermions. This can be achieved by attaching $2 \pi$ "statistical" flux to each vortex, introducing a Chern-Simons gauge field $A_{\mu}$. If we denote the Lagrangian in Eq. (2) as $\mathcal{L}_{\mathrm{v}}\left(\Psi, a_{\mu}, \alpha_{\mu}\right)$, the Lagrangian for the fermionized vortices (denoted as $\psi$ ) is

$$
\mathcal{L}_{\mathrm{fv}}=\mathcal{L}_{\mathrm{v}}(\psi, a-A, \alpha)+\frac{i}{4 \pi} A_{\mu} \epsilon_{\mu \nu \lambda} \partial_{\nu} A_{\lambda} .
$$

As recently argued, fermionization of vortices is expected to be an extremely good approximation due to the longranged intervortex interaction [16]. Indeed, by defining $a_{\mu}^{\prime}=a_{\mu}-A_{\mu}$, and then integrating over $A_{\mu}$, we absorb $A_{\mu}$ into $a_{\mu}$. One thereby obtains, $\mathcal{L}_{\mathrm{fv}}=\mathcal{L}_{\mathrm{v}}\left(\psi, a^{\prime}, \alpha\right)+$ $O\left(\partial^{3} a^{\prime 2}\right)$, where we have dropped terms less important than the Maxwell term present in $\mathcal{L}_{\mathrm{v}}$. Remarkably, the resulting theory of fermionized vortices has no ChernSimons term. The full Lagrangian, $\mathcal{L}_{\mathrm{fv}}+\mathcal{L}_{\mathrm{s}}+\mathcal{L}_{\text {int }}$, describes fermionic vortices and spinons interacting via a statistical interaction, and constitutes our two-fluid formulation of 2D superconductors in a field.

Generally, the fermionized vortices will see an effective average "dual" magnetic field with strength

$$
b_{\mathrm{fv}}=\pi\left\langle\hat{n}_{0}-\hat{n}_{\mathrm{f}}-2 \hat{N}_{\mathrm{v}}\right\rangle .
$$

The electrical charge density and the vortex density are externally determined conserved quantities. But now consider $\hat{n}_{\mathrm{f}}$ : as it stands, in addition to the conservation of total electrical charge, the total spinon (or electron) number, $N_{\mathrm{f}}=\int d \mathbf{x}\left\langle\hat{n}_{\mathrm{f}}\right\rangle$ is also conserved by $\mathcal{L}$. This additional global symmetry is present because we have dropped a term proportional to $\Delta$,

$$
\mathcal{L}_{\Delta}=\Delta\left[f_{\mathbf{r} \uparrow} f_{\mathbf{r} \downarrow} \mathcal{O}_{\alpha}^{\dagger}(\mathbf{r})+\text { H.c. }\right],
$$

where the operator $\mathcal{O}_{\alpha}^{\dagger}(\mathbf{r})$ inserts $2 \pi$ flux in $\epsilon_{i j} \partial_{i} \alpha_{j}$ at $\mathbf{r}$. This term originates microscopically from the term $e^{-i \phi} c c$ whereby a Cooper pair is created from two electrons. As we will later show, the term $\mathcal{L}_{\Delta}$ is perturbatively irrelevant in the vortex metal phase. Thus the conservation of $N_{\mathrm{f}}$ is an "emergent symmetry" not present microscopically. Although the term in Eq. (7) is irrelevant, it has an important role: it allows the spinon density to adjust, such that the total energy of the system with $\Delta=0$ is minimized. This condition then determines the spinon density $\hat{n}_{\mathrm{f}}$. It is possible that over some range of parameters the spinon density adjusts itself to make $b_{\mathrm{fv}}=0$ and fermionic vortices are natural variables. Another example is the limit of vanishing vortex mass, $m_{\mathrm{v}} \rightarrow 0$. In this case, the bosonic vortices "condense" and expel the flux $\left(b_{\mathrm{v}}=0\right)$, which sets $\left\langle\hat{n}_{\mathrm{f}}\right\rangle=n_{0}$, effectively "gluing" the charge back on to the spinons. Thus, one recovers an ordinary Fermi liquid of electrons. Alternately, this may be described as an integer quantum Hall state for fermionized vortices with $\nu_{\mathrm{v}}=1$.

Despite the average effective field in Eq. (6), the transverse force on a moving vortex is expected to be small in the disordered situations of interest - essentially due to the "normal" core, the local dual magnetic field in the vicinity of the vortex is small. In this case, we expect that the fermionized vortices can quantum diffuse, leading to a nonzero resistance at low temperatures. Below we explore the properties of the vortex metal phase. 
For transport properties, a semiclassical Boltzmann equation or even Drude theory is probably adequate. In the latter case, the statistics of the particles are not important, but it is essential that the statistical interaction between vortices and spinons is correctly incorporated. Specifically, when the spinons move they induce an electromotive force (EMF) on the vortices and vice versa. Similarly, an electrical charge current induces an EMF on the vortices. These effects can be summarized in the following transport equations, which describe the response of the system to an electrical current $\overrightarrow{\mathbf{J}}$ and to a thermal gradient $\nabla T$ :

$$
\begin{aligned}
& \overrightarrow{\mathbf{j}}_{\mathrm{v}}=\sigma_{\mathrm{v}} \cdot \hat{z} \times\left(\overrightarrow{\mathbf{j}}_{\mathrm{s}}-\overrightarrow{\mathbf{J}}\right)-\lambda_{\mathrm{v}} \nabla T ; \\
& \overrightarrow{\mathbf{j}}_{\mathrm{s}}=\sigma_{\mathrm{s}} \cdot \hat{z} \times \overrightarrow{\mathbf{j}}_{\mathrm{v}}-\lambda_{\mathrm{s}} \nabla T,
\end{aligned}
$$

where $\overrightarrow{\mathbf{j}}_{\mathrm{v} / \mathrm{s}}$ are vortex and spinon currents, respectively, and $\hat{z}$ is a unit vector normal to the film. Here, $\sigma_{\mathrm{v} / \mathrm{s}}$ and $\lambda_{\mathrm{v} / \mathrm{s}}$ are dimensionless transport "conductivities" of the bosonic vortices/spinons determining their current response to the EMF and thermal gradient. Generally these quantities are two-by-two matrices with off-diagonal components. The resistivity tensor of the fermionic vortices $\left(\sigma_{\mathrm{fv}}\right)^{-1}$ will differ from those of the bosonic ones by integer offdiagonal terms due to the Chern-Simons flux attachment. Thus, $\left(\sigma_{\mathrm{fv}}\right)^{-1}=\left(\sigma_{\mathrm{v}}\right)^{-1}+2 \pi \epsilon$, with $\epsilon$ the unit antisymmetric tensor $\left(\epsilon_{x y}=1\right)$. The physical electrochemical potential gradient is given by $\overrightarrow{\mathcal{E}}=\hat{z} \times \overrightarrow{\mathbf{j}}_{\mathrm{v}}$. Note that both the magnetic field and the electric field seen by the spinons are the same as the external ones. Thus the spinons respond to the external electromagnetic field as electrons. In the absence of a thermal gradient one can extract the (dimensionless) electrical sheet conductance matrix defined by, $J=\sigma \mathcal{E}$,

$$
\sigma=\left(\sigma_{\mathrm{v}}\right)^{-1}+\sigma_{\mathrm{s}}
$$

Thus the spinon and vortex contributions to the total conductivity simply add within this two-fluid theory. It is expected that the off-diagonal components of $\sigma_{\mathrm{v}}^{-1}$ and $\sigma_{\mathrm{s}}$ are both small compared to the diagonal component. Ignoring them, we obtain for the longitudinal resistance $R=\sigma_{\mathrm{v}}^{x x} /\left(1+\sigma_{\mathrm{v}}^{x x} \sigma_{\mathrm{s}}^{x x}\right)$.

Before exploring the vortex metal phase, we discuss the expected $(T=0)$ behavior of $\sigma_{\mathrm{v} / \mathrm{s}}$ as the strength of the external magnetic field is varied (Fig. 1). At low field the vortices are well separated and localized by impurities and the strong intervortex interaction, implying $\sigma_{\mathrm{v}}^{x x}=0$. This is the superconducting (vortex glass) phase with $R(T=$ $0)=0$. With increasing field and density, the vortices can delocalize at a field denoted by $B_{2}$ in Fig. 1, and will quantum diffuse with nonzero diagonal $\sigma_{\mathrm{v}}^{x x}$ and nonzero electrical resistance - this is the vortex metal. Finally, at large fields $\left(B_{3}\right)$ the bosonic vortices can condense giving $\sigma_{\mathrm{v}}^{x x}=\infty$ (the fermionized vortices form the $\nu=1$ quantum Hall state with $\sigma_{\mathrm{fv}}^{x x}=0$ ). This is the conventional Fermi liquid with $R=1 / \sigma_{\mathrm{s}}$. The spinon conductivity is likewise expected to be zero at very low magnetic fields,

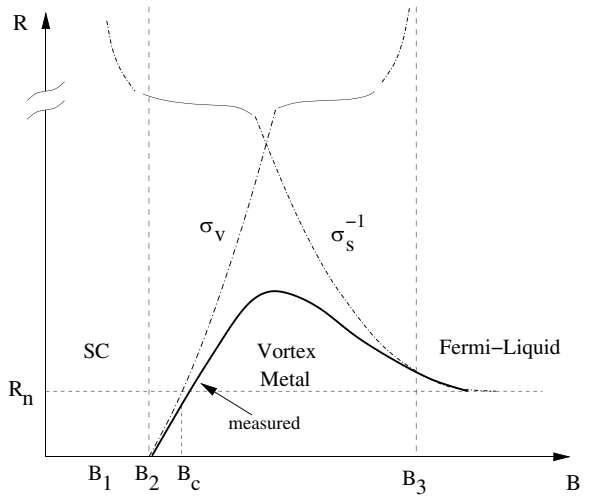

FIG. 1. Schematic $T=0$ phase diagram of amorphous superconducting films in the two-fluid model, as a function of an applied magnetic field. Dashed curves label the vortex conductivity and spinon resistivity, which determine, via Eq. (9), the electrical resistance (solid line). The vortex metal phase separates the low field superconductor $\left(B<B_{2}\right)$ from the high field Fermi liquid $\left(B>B_{3}\right)$.

since the spinons will predominantly be localized at the cores of the well separated vortices. At some field, $B_{1}$, spinons may become delocalized, and form an impurity band connecting vortices [17]. If this occurs with $\sigma_{\mathrm{v}}^{x x}=0$, this is a "superconducting thermal metal" phase. Finally, in the very high field limit, the spinon resistivity approaches the normal Fermi-liquid value, $R_{N}$.

For very disordered films with weakened superconductivity, the vortices will be especially mobile, and will condense at low magnetic fields, $B_{3} \ll H_{\mathrm{c} 2}$. With dilute vortices the spinon resistivity could be significantly larger than $R_{N}$ at $B \approx B_{3}$, and the films' electrical resistance will have a very large peak just below $B_{3}$. This behavior is consistent to that observed in such disordered samples.

Now consider the thermal conductivity $\boldsymbol{\kappa}^{x x}$. We assume that Fermi-liquid theory applies separately to both the fermionized vortices and spinons, then $\kappa^{x x}=T\left(\sigma_{\mathrm{fv}}^{x x}+\right.$ $\sigma_{\mathrm{s}}^{x x}$ ) (setting the Lorentz ratio to be 1). Thus the Lorentz ratio for the two-fluid vortex metal is $L=\kappa^{x x} /$ $\sigma^{x x} T=\left(\sigma_{\mathrm{s}}^{x x}+\sigma_{\mathrm{fv}}^{x x}\right) /\left[\sigma_{\mathrm{s}}^{x x}+\left(\sigma_{\mathrm{v}}^{x x}\right)^{-1}\right]$. This violation of the Wiedemann-Franz law is a striking signature of the nonFermi-liquid nature of the vortex metal. Notice that $L$ varies from zero to one as the magnetic field is tuned through the vortex metal phase from $B_{2}$ up to $B_{3}$. Thus, the vortex metal naturally interpolates between the superconducting and normal phases. As for the Nernst effect, $\mathcal{N}=$ $\mathcal{E}_{y} / \partial_{x} T$, with $\vec{J}=0$, ignoring small contributions from the off-diagonal conductivities we find, $\mathcal{N}=\left(\lambda_{\mathrm{v}}^{x x} / \sigma_{\mathrm{v}}^{x x}\right) R$. Within Fermi-liquid theory $\lambda_{\mathrm{v}}^{x x} \propto T \sigma_{\mathrm{fv}}^{x x}$, the Nernst signal is predicted to exhibit a nonmonotonic $B$-field dependence.

To address the Hall response, we need to consider nonzero off-diagonal conductivities. The Hall angle is then,

$$
\tan \theta_{\mathrm{H}}=\frac{\sigma_{\mathrm{v}}^{x y}+A \sigma_{\mathrm{s}}^{x y}}{\sigma_{\mathrm{v}}^{x x}+A \sigma_{\mathrm{s}}^{x x}} ; \quad A=\left(\sigma_{\mathrm{v}}^{x x}\right)^{2}+\left(\sigma_{\mathrm{v}}^{x y}\right)^{2} .
$$

Another quantity of interest is the Nernst angle. Again 
ignoring small off-diagonal contributions, we get $\tan \theta_{\mathrm{N}}=$ $\lambda_{\mathrm{s}}^{x x} \sigma_{\mathrm{v}}^{x x} / \lambda_{\mathrm{v}}^{x x}$. Thus the transport angles $\theta_{\mathrm{H}}, \theta_{\mathrm{N}}$ differ in the vortex metal.

Next we consider the specific heat $C(T)$ in the vortex metal at low $T$. Naively, the vortex and spinon Fermi seas would give the usual $C \sim T$ behavior. But with gauge fluctuations, and within RPA, we obtain $C(T) \sim T \ln (1 / T)$.

Let us now discuss tunneling properties. Electron tunneling from a metallic tip is expected to be similar to that in a conventional 2D metal due to the gapless electronlike quasiparticles present in the vortex metal state. With a superconducting tip placed above the film, one can measure the Cooper-pair tunneling conductance into the vortex metal [18]. The conductance $G=G_{\mathrm{A}}+G_{\mathrm{cp}}$ has two parts: $G_{\mathrm{A}}$ is due to simultaneous tunneling of two electrons, which is equivalent to the Andreev reflection of an incident hole, and $G_{\mathrm{cp}}$ is due to tunneling of Cooper pairs that get absorbed in the Cooper-pair fluid. Whereas $G_{\mathrm{A}}$ measures the local density of states of the gapless quasiparticles and is almost temperature independent, $G_{\mathrm{cp}}$ depends strongly on temperature. $G_{\mathrm{cp}}$ is given by the Kubo formula, $G_{\mathrm{cp}} \propto$ $i \int d t t\langle[I(t), I(0)]\rangle$. With the tip at $\mathbf{x}=\mathbf{0}$, the Cooper-pair current operator is $I=2 e J \sin \phi_{0}$. First, we evaluate the imaginary time correlator $C(\tau)=\left\langle e^{i \phi_{0}(\tau)} e^{-i \phi_{0}(0)}\right\rangle$. The density of Cooper pairs is the "dual magnetic field," $b=$ $\epsilon_{i j} \partial_{i} a_{j}$ and the Cooper-pair current is $\hat{z} \times \mathbf{e}$ with "electric field," $\mathbf{e}=-\nabla a_{0}-\partial_{0} \mathbf{a} . C(\tau)$ describes the process of inserting a monopole at $\mathbf{x}=\mathbf{0}$ and removing it at a later time $\tau$. The action of this monopole configuration determines $C(\tau)$; we calculate it within a quadratic action for the gauge field. Since the spinons do not affect the dominant large $\tau$ behavior, we focus on the action due to vortices. Integrating them out within RPA, we obtain $S_{0}=$ $\frac{T}{2} \Sigma_{\omega_{n}} \int \frac{d^{2} \mathbf{q}}{(2 \pi)^{2}}\left[\varepsilon_{\alpha \beta} e_{\alpha} e_{\beta}+\mu b^{2}\right]+S_{\lambda} . S_{0}$ is expressed using the gauge invariant fields e and $b$. Here $S_{\lambda}=i \int d x \lambda(x) \times$ $\left[\nabla \times \mathbf{e}+\partial_{0} b-\rho_{\mathrm{m}}(x)\right]$ enforces the dual Maxwell equation, with $\lambda(x)$ a Lagrange multiplier and $\rho_{\mathrm{m}}(x)$ the density of monopoles; we set $\rho_{\mathrm{m}}\left(x_{0}, \mathbf{r}\right)=\delta\left(x_{0}\right) \delta(\mathbf{r})$ $-\delta\left(x_{0}-\tau\right) \delta(\mathbf{r})$. In $S_{0}, \varepsilon_{\alpha \beta}$ is the dielectric constant determined by the diffusive fermionic vortices, with transverse part $\varepsilon^{(\mathrm{tr})}=\sigma_{\mathrm{v}} /\left|\omega_{n}\right|$. The permeability $\mu=\chi_{\mathrm{v}}$ is the vortex orbital "magnetic" susceptibility. Thus the action of the monopole configuration is $S(\tau)=\frac{\sigma_{v}}{2} \ln ^{2}\left(\tau / \tau_{0}\right)$, with $\tau_{0}^{-1}$ the scattering rate of the diffusive vortices. Inserting $C(\tau) \propto \exp [-S(\tau)]$ into the Kubo formula,

$$
G_{\mathrm{cp}}(T) \propto T^{-2} \exp \left[-\frac{\sigma_{\mathrm{v}}}{2} \ln ^{2}\left(T \tau_{0}\right)\right] .
$$

Note that the Andreev part of the conductance, $G_{\mathrm{A}}$, which describes two electron tunneling, adds a $T$-independent contribution to $G$, which traces $\sigma_{\mathrm{s}}: G_{\mathrm{A}} \propto \sigma_{\mathrm{s}}^{2}$. Near the superconducting transition, $B_{2}, G_{\mathrm{cp}}$ is expected to dominate the tunneling since $\sigma_{\mathrm{v}}$ is small. Particularly, when vortices are localized $G_{\mathrm{cp}}$ diverges, which corresponds to the appearance of the Josephson effect. As the magnetic field, $\sigma_{\mathrm{v}}$, and $\sigma_{\mathrm{s}}$ grow, $G_{\mathrm{A}}$ will increase and the temperature dependence of $G$ gets suppressed.

Using the above results, we can now determine that $\mathcal{L}_{\Delta}$ in Eq. (7) is perturbatively irrelevant within RPA in the vortex metal. This is inferred from the two-point correlation function of $f_{\uparrow} f_{\downarrow} \mathcal{O}_{\alpha} \sim e^{i \phi} c_{\uparrow} c_{\downarrow}$ evaluated at $\Delta=0$. Factorizing $e^{i \phi} c_{\uparrow} c_{\downarrow}$, the electrons contribute a power law: $\left\langle c_{\alpha}(\tau) c_{\alpha}^{\dagger}(0)\right\rangle \sim \tau^{-1}$, and also $C(\tau)=\left\langle e^{i \phi_{0}(\tau)} \times e^{-i \phi_{0}(0)}\right\rangle \propto$ $\exp \left[-\sigma_{\mathrm{v}} \ln ^{2}\left(\tau / \tau_{0}\right)\right]$. The product of the two decays faster than any power law, rendering $\mathcal{L}_{\Delta}$ irrelevant.

In this Letter we introduced a new two-fluid description for amorphous superconducting films in a magnetic field. Fermionization of the field-induced vortices allowed us to access a resistive quantum-liquid phase that naturally interpolates between the superconductor and the normal metal. Anomalous thermoelectric transport and tunneling behavior were predicted in the vortex metal.

It is a pleasure to thank A. Auerbach, L. Balents, A. Kapitulnik, S. Kivelson, A. I. Larkin, O. Motrunich, Y. Oreg, and D. Shahar for discussions on this work. This work was generously supported by the David and Lucile Packard foundation (V. G.) and by the National Science Foundation through Grant Nos. PHY99-07949 (V.G., G. R., and M.P.A.F.), DMR-0210790 (M.P.A.F.), and DMR-0308945 (T.S.). T.S. also acknowledges funding from the NEC Corporation, the Alfred P. Sloan Foundation, and The Research Corporation.

[1] D. B. Haviland, Y. Liu, and A. M. Goldman, Phys. Rev. Lett. 62, 2180 (1989).

[2] A. F. Hebard and M. A. Paalanen, Phys. Rev. Lett. 65, 927 (1990).

[3] V.F. Gantmakher et al., JETP Lett. 68, 363 (1998).

[4] M.P. A. Fisher, Phys. Rev. Lett. 65, 923 (1990).

[5] N. Mason and A. Kapitulnik, Phys. Rev. Lett. 82, 5341 (1999).

[6] N. Mason and A. Kapitulnik, Phys. Rev. B 64, 060504 (2001).

[7] M. A. Paalanen, A. F. Hebard, and R. R. Ruel, Phys. Rev. Lett. 69, 1604 (1992).

[8] G. Sambandamurthy et al., Phys. Rev. Lett. 92, 107005 (2004).

[9] M. A. Steiner, G. Boebinger, and A. Kapitulnik, Phys. Rev. Lett. 94, 107008 (2005)

[10] D. Dalidovic and P. Phillips, Phys. Rev. B 64, 052507 (2001).

[11] A. Kapitulnik et al., Phys. Rev. B 63, 125322 (2001).

[12] M. V. Feigelman et al., Phys. Rev. B 48, 16641 (1993).

[13] T. Senthil and M. P. A. Fisher, Phys. Rev. B 62, 7850 (2000).

[14] L. Balents and M. P. A. Fisher, Phys. Rev. B 71, 085119 (2005).

[15] N. Read, Phys. Rev. B 58, 16262 (1998).

[16] J. Alicea et al., cond-mat/0503399.

[17] S. Vishveshwara, T. Senthil, and M.P. A. Fisher, Phys. Rev. B 61, 6966 (2000).

[18] O. Naaman, W. Teizer, and R. Dynes, Phys. Rev. Lett. 87, 097004 (2001). 